Research Article

\title{
Test-Retest Reliability of the Coronary Heart Disease Damp Phlegm and Blood Stasis Pattern Questionnaire: Results from a Multicenter Clinical Trial
}

\author{
Ge Fang $\mathbb{D},{ }^{1}$ Yaxin Wang $\mathbb{D}^{2},{ }^{2}$ Zhenqian Yan $\mathbb{D}^{2},{ }^{2}$ Xiaowen Zhou $\mathbb{D}^{2},{ }^{2}$ Xingyu Fan $\mathbb{D},{ }^{1}$ \\ Xiaoqian Liao $\mathbb{D},{ }^{1}$ Zhixi Hu $\mathbb{D},^{3}$ and Xiantao Li $\mathbb{D}^{2}$ \\ ${ }^{1}$ College of Traditional Chinese Medicine, Hunan University of Chinese Medicine, Changsha 410208, China \\ ${ }^{2}$ School of Basic Medical Science, Guangzhou University of Chinese Medicine, Guangzhou 510006, China \\ ${ }^{3}$ Institute of Chinese Medicine Diagnosis, Hunan University of Chinese Medicine, Changsha 410208, China
}

Correspondence should be addressed to Zhixi Hu; 003405@hnucm.edu.cn and Xiantao Li; lixiantao@gzucm.edu.cn

Received 31 July 2021; Revised 20 October 2021; Accepted 21 October 2021; Published 16 November 2021

Academic Editor: Mohammad Hashem Hashempur Copyright $\odot 2021 \mathrm{Ge}$ Fang et al. This is an open access article distributed under the Creative Commons Attribution License, which
permits unrestricted use, distribution, and reproduction in any medium, provided the original work is properly cited.

Background. Damp phlegm and blood stasis pattern (DPBSP) is the main pattern in coronary heart disease (CHD) patients. To quantify and standardize the diagnosis of DPBSP, questionnaires are usually administered. The CHD Damp Phlegm and Blood Stasis Pattern Questionnaire (CHD-DPBSPQ) is the standard metric for measuring CHD-DPBSP signs and symptoms in practice and clinical research. The CHD-DPBSPQ has moderate diagnostic efficiency, as evidenced by its receiver operating characteristic curves. Furthermore, and high reliability and validity have been shown in some studies but not in a multicenter clinical trial. Our purpose was to evaluate the test-retest reliability of a proprietary CHD-DPBSPQ. Methods. The CHD-DPBSPQ uses a standard procedure for measuring symptoms. The (interrater) reliability and validity of this questionnaire have been previously studied. Here, we evaluated the test interval and weighted kappa value of items of testretest (intrarater) reliability of the CHD-DPBSPQ. The test-retest reliability was evaluated by the intraclass correlation coefficient (ICC) for the total CHD-DPBSPQ score and the phlegm domain and blood stasis domain scores. Weighted kappa statistics were calculated for the individual CHD-DPBSPQ items. Results. Using the CHD-DPBSPQ, 79 patients with latestage CHD who were participating in a multicenter clinical trial were assessed twice. The ICCs for the CHD-DPBSPQ score were as follows: 0.827 for the total CHD-DPBSPQ, 0.778 for the phlegm domain score, and 0.828 for the blood stasis domain score. The reliability was slightly better in patients whose test interval was $\leq 14$ days. The weighted kappa values of individual items showed moderate consistency. Conclusions. The CHD-DPBSPQ was found to have excellent test-retest reliability in this sample of patients.

\section{Introduction}

Coronary heart disease (CHD) has been the main cause of mortality and disability in both developed and developing countries in the last 20 years [1]. According to the Report on Cardiovascular Health and Diseases in China 2019, there were approximately 11 million CHD patients who were more than 15 years old in China [2]. In Western countries, CHD accounted for about around a third of all the deaths in people aged above 35 , although CHD mortality has gradually declined [3]. This may be related to increased serum cholesterol levels caused by sex, age, hyperlipidemia, hypertension, diabetes, obesity, smoking, and other changes [4-8]. The prevalence of CHD is expected to continue to increase with contemporary lifestyles. Therefore, we need to rapidly strengthen the diagnostic criteria of $\mathrm{CHD}$ and provide better treatment for clinical patients.

Epidemiological investigations of the CHD syndrome have shown that the distributional characteristics are mainly blood stasis and phlegm turbidity. For example, Mao et al. found that the main syndrome characteristics of CHD from 1970 to 2010 were blood stasis, phlegm turbidity, and qi 
stagnation [9]. Wang et al. found that blood stasis, phlegm turbidity, and qi deficiency were the main syndrome elements of CHD by analyzing 115 cases of CHD diagnosed and treated by famous doctors [10].

The Coronary Heart Disease Damp Phlegm and Blood Stasis Pattern Questionnaire (CHD-DPBSPQ) is the standard metric for measuring CHD-DPBSP signs and symptoms in practice and clinical research [11-13]. The CHDDPBSPQ was published in 2019 by combining an assessment of the damp phlegm pattern and blood stasis pattern in CHD Patients [14]. In other words, the CHD-DPBSPQ consists of two main domains that assess turbid phlegm and blood stasis [15]. According to the epidemiological investigation of Traditional Chinese Medicine (TCM) Syndromes of CHD in China from 1990 to 2020, the prevalence of blood stasis syndrome and phlegm turbidity syndrome amounted to $64.2 \%$ and $37.8 \%$, respectively, and the two ranked in the top three syndromes [16].

TCM syndromes can be constructed into a set of standards and issued in the form of scales. Several studies have investigated the structure and metric properties of the CHD-DPBSPQ. The CHD-DPBSPQ has been shown to have a moderate diagnostic efficiency based on its receiver operating characteristic curves [12]. Some studies have shown that the CHD-DPBSPQ has high reliability and validity but the metric has yet to be assessed in a multicenter clinical trial [14]. The study's aim was to present research aimed at the test-retest reliability of the CHD-DPBSPQ in a multicenter clinical trial comprising CHD patients.

\section{Materials and Methods}

2.1. Subjects. The researchers had to identify patients who could participate in the questionnaire again to ensure that the number of respondents in the two surveys was five times the number of items in the questionnaire. In accordance with the study protocol, the inclusion criteria were as follows: the subjects were aged 18 years or above, provided informed consent for participation, had been diagnosed with CHD according to the guidelines $[17,18]$, and had been diagnosed with DPBSP by two experienced experts [19]. The exclusion criteria were as follows: patients with unstable angina and a diagnosis of diseases or syndromes other than CHD.

2.2. Raters. The raters were the main researchers. The raters were all experts in TCM or integrated Chinese and Western medicine in the treatment of cardiovascular diseases, with at least 20 years of clinical experience. All the raters explained the questionnaire to each patient before any evaluations were performed.

2.3. Cross-Sectional Validation of the Questionnaire. The questionnaire was validated in a cross-sectional, multicenter, observational, descriptive study that followed a test-retest design. The study was approved by the Research Ethics Committee of Tianjin University of Traditional Chinese Medicine (no. TJUTCMEC2015000). The construction definition, item generation, selection reliability, and validity of the CHD-DPBSPQ were performed according to standard procedures [20-22]. Next, we assessed the two domains in the questionnaire: the phlegm domain (chest distress, sleepiness, physical heaviness, obesity, sticky mouth, abdominal fullness, anorexia, greasy tongue fur, and slippery pulse) and the blood stasis domain (chest pain, cyanotic lips, dim complexion, dark purple tongue, petechiae or ecchymosis on the tongue, and sublingual vein cyanosis). The items were rated according to four response options: $0=$ none, $1=$ mild, $2=$ moderate, and $3=$ severe. Higher scores indicate a greater severity of the symptom in question.

2.4. Statistical Analysis. For the statistical analysis, SPSS version 25.0 (IBM SPSS Statistics for Windows, IBM Corp., Armonk, NY) and SAS software 9.4 (SAS Institute, Cary, NC) were used. The test-retest reliabilities of the different domains of the CHD-DPBSPQ were estimated by the intraclass correlation coefficient (ICC) [23]; the 95\% confidence interval was also calculated. The test-retest reliability was estimated separately for the subdomains of subjects as defined by the number of days (with the 14th day as the dividing line). The test-retest reliability for single items was assessed by the weighted kappa statistics [24].

\section{Results}

In our preliminary research, the data of 729 inpatients (from eight hospitals in the period between 2016 and 2018) were collected and screened from a doctor according to the aforementioned inclusion and exclusion criteria. Ultimately, two experienced CHD experts in TCM or integrated Chinese and Western medicine diagnostic patterns collected 79 inpatient data, which comprised the research sample. The specific data processing flow diagram that was designed is shown in Figure 1. The clinical characteristics of $79 \mathrm{CHD}$ subjects with DPBSP (mean age: 65 years; most were males) were collected from different hospitals in China for the testretest study (Table 1). All the patients had comorbidities, were of the Han ethnicity, and were married. The course of the disease was generally within 30 days. Patients with different severities of illness had different questionnaire scores.

Figure 2 shows the frequency of the four response options of the items in the first and the second scale tests. The ICCs for the test-retest reliability and related $95 \%$ confidence intervals are shown in Table 2. The ICCs were 0.827 for the total CHD-DPBSPQ, 0.778 for the phlegm domain, and 0.828 for the blood stasis domain. The ICCs for the patients whose test interval was $>14$ days were lower than those of the patients whose test interval was $\leq 14$ days. The weighted kappa value of items was $\geq 0.4$ for most items except abdominal fullness and greasy tongue fur, which were 0.3372 and 0.2238 , respectively (Table 3 ).

\section{Discussion}

The CHD-DPBSPQ was developed and evaluated according to a set of standard procedures and then issued by the China 


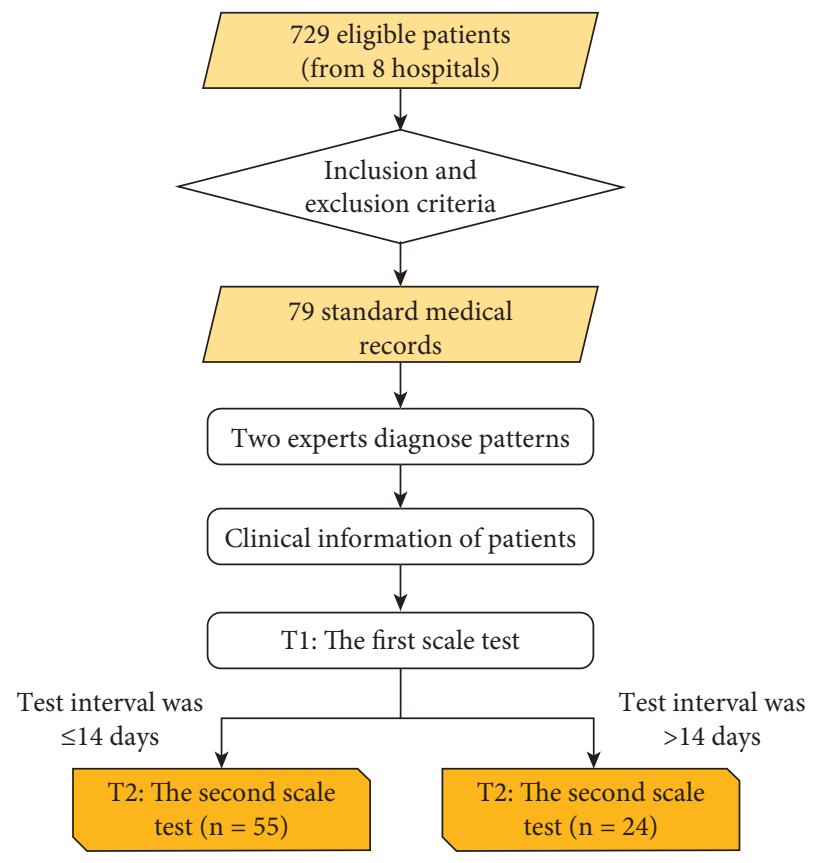

Figure 1: A flow diagram for the patients.

Association of Chinese Medicine (CACM). To date, there has been no report of a cross-sectional study of TCM syndromes combined with a joint investigation of patients from multiple hospitals.

Our results showed that the total CHD-DPBSPQ and blood stasis domain had excellent test-retest reliabilities; the symptom-based subscales also showed considerably good reliability. However, the test-retest reliability of the phlegm domain was somewhat lower than that of the blood stasis domain of the CHD-DPBSPQ. This result may be because of the different characteristics of people in different regions, which reflect the so-called "treating the disease according to the individual condition" and "treating the disease according to the environment" aspects of TCM. However, the test-retest reliability of the phlegm domain was close to 0.75 , which is the lower-limit indicator of good diagnostic tests. The ICCs were slightly better for patients with test-retest intervals $\leq 14$ days than for those of patients with intervals $>14$ days. This finding is consistent with Andrew's report on the time interval [25]. The lower test-retest reliability of every CHDDPBSPQ item can be explained by the fact that our sample of patients with $>14$ days of intervals generally achieved low scores on some items. Sleepiness (0.5602), physical heaviness (0.5194), sticky mouth (0.4662), anorexia (0.4256), chest pain (0.4332), dark purple tongue (0.4121), petechiae, or ecchymosis of the tongue $(0.5298)$ items had weighted kappa values that were $<0.60$. Fleiss [26] suggested that the ICCs of items or questionnaires of $<0.4$ indicate a "poor" reliability. This result may be due to the particularity and clinical symptoms of TCM syndromes. The determination of a TCM syndrome is based on a series of syndrome groups. In clinical practice, even if patients show the damp phlegm and blood stasis
TABLE 1: Characteristics of the patients with CHD-DPBSP* $(\mathrm{n}=79)$.

\begin{tabular}{lc}
\hline Variable & \\
Age (year) & $67 \pm 12.1$ \\
Male (\%) & $47(60.5 \%)$ \\
Comorbidities (\%) & $68(87.2 \%)$ \\
Han ethnicity (\%) & $79(100 \%)$ \\
Marriage (\%) & $79(100 \%)$ \\
Years since CHD diagnosis (days) & \\
$\quad \leq 30$ & $40(50.6 \%)$ \\
$\leq 60$ & $12(15.2 \%)$ \\
$\leq 90$ & $7(8.9 \%)$ \\
$>90$ & $20(25.3 \%)$ \\
CHD-DPBSPQ (possible range) & \\
Total (0-45) & $18.2 \pm 9.2$ \\
Phlegm (0-27) & $10.2 \pm 5.4$ \\
Blood stasis (0-18) & $8.0 \pm 4.6$ \\
\hline
\end{tabular}

${ }^{*}$ CHD-DPBSP was diagnosed according to the International Statistical Classification of Diseases and Related Health Problems 10th Revision (ICD10).

pattern, the symptoms of these patients are not completely consistent. For example, "greasy fur" is common in China for people who are from the south but rare for those from the north.

Our study aimed to validate the test-retest reliability of the CHD-DPBSPQ in patients at multiple treatment centers in China. However, the results showed individual differences in the degree of the importance of the different items in the CHD-DPBSP. Even if the individual items of domains show low scores, these results are not necessarily reflected in the total CHD-DPBSPQ score. The CHD-DPBS syndrome is divided into two stages: phlegm in the early stage and blood stasis in the late stage. The average age of our patients was approximately 66 years, at which it is common for late-stage 


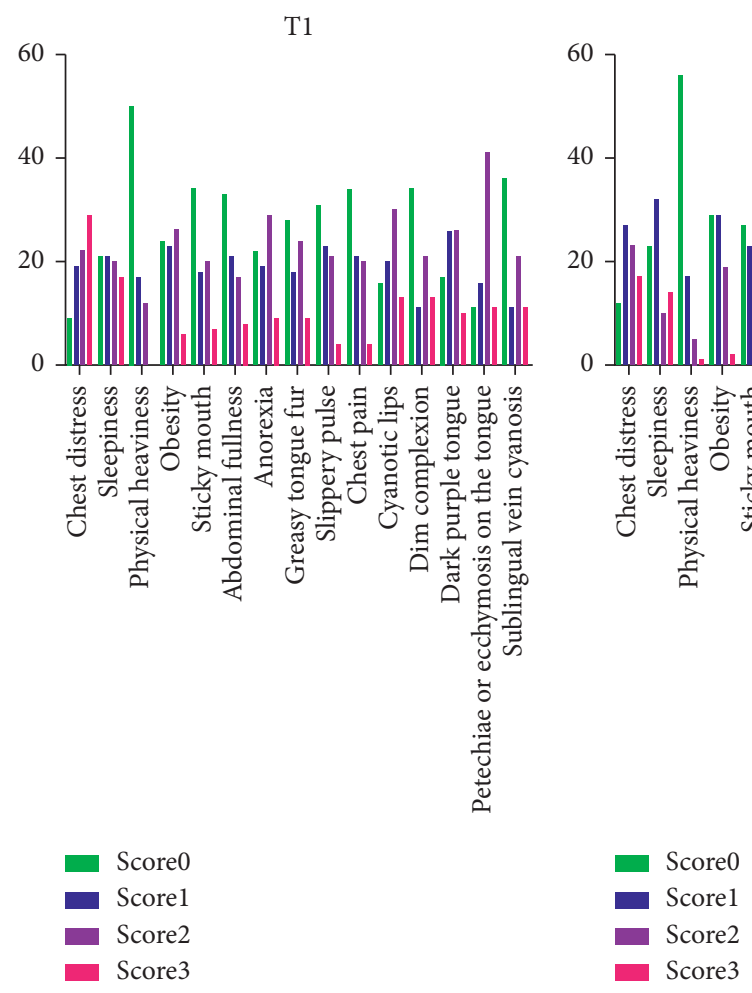

(a)

(b)

FIGURE 2: Frequency of the responses in the two-round scale test for each item. T1: the first scale test; T2: the second scale test.

TABLE 2: ICCs for the total CHD-DPBSPQ and subscales for all patients, those reassessed $\leq 14$ days, and those reassessed $>14$ days after screening*.

\begin{tabular}{lcrr}
\hline & All patients & \multicolumn{2}{c}{ Time of test-retest reliability } \\
& & 14 or 14 fewer days & More than 14 days \\
\hline Total CHD-DPBSPQ & $0.827(0.729,0.889)$ & $0.897(0.824,0.940)$ & $0.802(0.542,0.914)$ \\
Phlegm domain & $0.778(0.653,0.858)$ & $0.850(0.742,0.912)$ & $0.734(0.385,0.885)$ \\
Blood stasis domain & $0.828(0.732,0.890)$ & $0.896(0.821,0.939)$ & $0.808(0.557,0.917)$ \\
\hline
\end{tabular}

${ }^{*} 95 \%$ confidence intervals for the ICCs are given in brackets.

TABLE 3: Weighted kappa values for all CHD-DPBSPQ items*.

\begin{tabular}{lr}
\hline Item & Weighted kappa \\
\hline Phlegm domain & \\
Chest distress & $0.3856(0.2478,0.5233)$ \\
Sleepiness & $0.5602(0.4060,0.7144)$ \\
Physical heaviness & $0.5194(0.3850,0.6537)$ \\
Obesity & $0.6609(0.5330,0.7889)$ \\
Sticky mouth & $0.4662(0.3255,0.6069)$ \\
Abdominal fullness & $0.3372(0.1784,0.4960)$ \\
Anorexia & $0.4256(0.2660,0.5853)$ \\
Greasy tongue fur & $0.2238(0.0781,0.3696)$ \\
Slippery pulse & $0.3920(0.2338,0.5501)$ \\
Blood stasis domain & \\
Chest pain & \\
Cyanotic lips & $0.4332(0.2773,0.5890)$ \\
Dim complexion & $0.6230(0.4992,0.7468)$ \\
Dark purple tongue & $0.6094(0.4819,0.7369)$ \\
Petechiae or ecchymosis on the tongue & $0.4121(0.2655,0.5587)$ \\
Sublingual vein cyanosis & $0.5298(0.3875,0.6720)$ \\
\hline
\end{tabular}

*95\% confidence intervals for the weighted kappa statistics are given in brackets. 
CHD patients to have "blood stasis" as the main stage, and the "phlegm" syndrome score is low. Deng [27] stated that phlegm is the initial stage of blood stasis, which further develops into blood stasis, which confirms the conclusion of the questionnaire. In general, the reliability score of the entire questionnaire was 0.827 , which was $>0.75$, indicating that slight internal changes would not affect the conclusions drawn from the questionnaire.

In particular, one limitation of our study was the lack of early CHD-DPBSP patients. Thus, future studies should investigate patients from aged 18-66 years. Owing to medication effects, a substantial short-term variability in the CHD-DPBSPQ scores should be expected. Because this investigation comprised the basic work of diagnostic research, the present study lacks the "prescription-syndrome correspondence" to evaluate the reactive evidence of the diagnostic criteria of DPBSP in CHD. That is, we only selected CHD patients with DPBSP for measurement and excluded those DPBSP.

Nevertheless, our investigation has some strengths which are as follows. First, we assessed 79 patients to evaluate the test-retest reliability, which met the requirements of a sample size of $>20$ participants [28]. Second, the patients were from multiple centers in China. Third, our raters were all CHD clinical experts who were considerably familiar with the CHD-DPBSPQ. Finally, few reports exist on the test-retest reliability of the CHDDPBSPQ; thus, the present research adds to the literature in this regard.

\section{Conclusions}

The CHD-DPBSPQ was found to be reliable and can be recommended for evaluations of CHD. The present study also showed that the CHD-DPBSPQ is a valid instrument for measuring the phlegm domain and blood stasis domain scores. The establishment of DPBSP provides a standard template for determining the test-retest reliability of TCM syndrome types and establishes a foundation for achieving TCM syndrome standardization.

\section{Abbreviations}

CHD- Coronary Heart Disease Damp Phlegm and DPBSPQ: Blood Stasis Pattern Questionnaire

ICC: Intraclass correlation coefficient

CHD: $\quad$ Coronary heart disease

DPBSP: $\quad$ Damp phlegm and blood stasis pattern

TCM: $\quad$ Traditional Chinese medicine

ICD-10: The International Statistical Classification of Diseases and Related Health Problems $10^{\text {th }}$ Revision

CACM: $\quad$ China Association of Chinese Medicine.

\section{Data Availability}

The datasets used and/or analyzed during the current study are available from the corresponding author on reasonable request.

\section{Additional Points}

Trial registration: ChiCTR-OOC-15006599, registered on June 16, 2015, http://www.chictr.org.cn/showproj.aspx? proj $=11233$.

\section{Ethical Approval}

The Research Ethics Committee of Tianjin University of Traditional Chinese Medicine provided approval for this study (no. TJUTCMEC2015000).

\section{Consent}

All patients provided informed consent.

\section{Conflicts of Interest}

The authors declare no conflicts of interest.

\section{Authors' Contributions}

Ge Fang participated in the study design, analyzed the data, and drafted and revised the manuscript. Yaxin Wang and Zhenqian Yan participated in the study design. Xiaowen Zhou collected and checked the data. Xingyu Fan and Xiaoqian Liao analyzed the data. Zhixi $\mathrm{Hu}$ was a major contributor in data collection and manuscript revision. Xiantao Li was a major contributor to the study conception and to manuscript drafting. All authors read and approved the final manuscript.

\section{Acknowledgments}

The authors thank the following teams for their valuable contributions: the Wu Bin Research Team at Hubei Provincial Hospital of Traditional Chinese Medicine, Yu Chunquan Research Team at Tianjin University of Traditional Chinese Medicine, Yang Xiaobo Research Team at Guangdong Provincial Hospital of Traditional Chinese Medicine, Dai Guohua Research Team of the Affiliated Hospital of Shandong University of Chinese Medicine, Zheng Guoqing Research Team at the Second Affiliated Hospital of Wenzhou Medical University, Qian Weidong Research Team of Changzhou City Hospital of Traditional Chinese Medicine, and Sun Gang Research Team at Guizhou Provincial Hospital of Traditional Chinese Medicine. This research was funded by the Key Areas of Research and Development Plan of Guangdong Province (No. 2020B1111100001) and Natural Science Foundation of Guangdong Province (No. 2020A1515010725).

\section{References}

[1] WHO, WHO Reveals Leading Causes of Death and Disability Worldwide: 2000-2019, In World Health Organization, Geneva, Switzerland, 2020, https://www.who.int/zh/news/ item/09-12-2020-who-reveals-leading-causes-of-death-anddisability-worldwide-2000-2019. 
[2] Z. W. Wang and S. S. Hu, "Interpretation of report on cardiovascular Health and diseases in China 2019," Chinese Journal of Cardiology, vol. 25, no. 5, pp. 401-410, 2020.

[3] S. Stolpe, B. Kowall, and A. Stang, "Decline of coronary heart disease mortality is strongly effected by changing patterns of underlying causes of death: an analysis of mortality data from 27 countries of the WHO European region 2000 and 2013," European Journal of Epidemiology, vol. 36, no. 1, pp. 57-68, 2021.

[4] M. H. Chiu, B. Heydari, Z. Batulan, N. Maarouf, V. Subramanya, and K. Schenck-Gustafsson, "Coronary artery disease in post-menopausal women: are there appropriate means of assessment?" Clinical Science, vol. 132, no. 17, pp. 1937-1952, 2018.

[5] G. W. Mikhail, "Coronary heart disease in women," $B M J$, vol. 331, no. 7515, pp. 467-468, 2005.

[6] M. V. Madhavan, B. J. Gersh, K. P. Alexander, C. B. Granger, and G. W. Stone, "Coronary artery disease in patients $\geq 80$ years of age," Journal of the American College of Cardiology, vol. 71, no. 18, pp. 2015-2040, 2018.

[7] S. Abram, A. M. Arruda-Olson, C. G. Scott et al., "Frequency, predictors, and implications of abnormal blood pressure responses during dobutamine stress echocardiography," Circulation. Cardiovascular imaging, vol. 10, no. 4, Article ID e005444, 2017.

[8] F. Liang and Y. Wang, "Coronary heart disease and atrial fibrillation: a vicious cycle," American Journal of Physiology. Heart and Circulatory Physiology, vol. 320, no. 1, pp. H1-H12, 2021.

[9] J. Y. Mao, Z. C. Niu, and B. L. Zhang, "Literature analysis of studies on the TCM syndromes of coronary heart disease in the recent 40 years," Journal of Traditional Chinese Medicine, vol. 52, no. 11, pp. 958-961, 2011.

[10] J. Wang, R. Wu, and X. Z. Zhou, "Syndrome factors based on SVM from coronary heart disease treated by prominent TCM doctors," Journal of Beijing University of Traditional Chinese Medicine, vol. 31, no. 8, pp. 540-543, 2008.

[11] X.-q. Liu, D.-h. Peng, Y.-p. Wang et al., "Diagnostic accuracy of Chinese medicine diagnosis scale of phlegm and blood stasis syndrome in coronary heart disease: a study protocol," Chinese Journal of Integrative Medicine, vol. 25, no. 7, pp. 515-520, 2019.

[12] X. Zhou, X. T. Li, X. Q. Liu, B. Wang, and G. Fang, "Assessment of intermingled phlegm and blood stasis syndrome in coronary heart disease: development of a diagnostic scale," Evidence Based Complementary and Alternative Medicine, vol. 2018, Article ID 4683431, 6 pages, 2018.

[13] Q. Ren, X.-w. Zhou, M.-y. He et al., "A quantitative diagnostic method for phlegm and blood stasis syndrome in coronary heart disease using tongue, face, and pulse indexes: an exploratory pilot study," Journal of Alternative \& Complementary Medicine, vol. 26, no. 8, pp. 729-737, 2020.

[14] G. Fang, L. L. Zhang, Q. Ren, X. W. Zhou, B. Wang, and $\mathrm{X}$. Zhou, "Development of a diagnostic questionnaire for damp phlegm pattern and blood stasis pattern in coronary heart disease patients (CHD-DPBSPQ)," Evidence Based Complementary and Alternative Medicine, vol. 2019, Article ID 6856085, 2019.

[15] J. Q. Hu, C. C. Wang, F. Duan, X. T. Li, R. Liu, and W. M. Xu, "Study on macrocosmic diagnostic criteria for coronary heart disease with intermingled phlegm- blood stasis syndrome," Zhongguo Zhong Xi Yi Jie He Za Zhi, vol. 36, no. 10, pp. 1164-1168, 2016.
[16] C. C. Wang, S. Wu, L. J. Jiang, W. M. Xu, X. Tong, and Y. R. Cai, "Research situation of traditional Chinese medicine clinical epidemiological investigation of coronary heart disease in China from 1990 to 2020," Chinese Journal of Basic Medicine in Traditional Chinese Medicine, vol. 26, no. 12, pp. 1883-1893, 2020.

[17] G. N. Levine, E. R. Bates, J. A. Bittl et al., "2016 ACC/AHA guideline focused update on duration of dual antiplatelet therapy in patients with coronary artery disease," Journal of the American College of Cardiology, vol. 68, no. 10, pp. 1082-1115, 2016.

[18] "Guideline for diagnosis and treatment of patients with chronic stable angina," Zhonghua Xinxueguanbing Zazhi, vol. 35, no. 3, pp. 195-206, 2007.

[19] Z. Wang, X.-H. Zhou, and M. Wang, "Evaluation of diagnostic accuracy in detecting ordered symptom statuses without a gold standard," Biostatistics, vol. 12, no. 3, pp. 567-581, 2011.

[20] F.-b. Liu, X.-l. Chen, L. Guo, and X.-b. Liu, "Evaluation of a scale of patient-reported outcomes for the assessment of myasthenia gravis patients in China," Chinese Journal of Integrative Medicine, vol. 18, no. 10, pp. 737-745, 2012.

[21] Q. Guo and Q. Chen, "Standardization of syndrome differentiation defined by traditional Chinese medicine in operative breast cancer: a modified delphi study," Scientific World Journal, vol. 2015, Article ID 820436, 2015.

[22] R. Q. Chen, C. M. Wong, and T. H. Lam, "Construction of a traditional Chinese medicine syndrome-specific outcome measure: the Kidney Deficiency Syndrome Questionnaire (KDSQ)," BMC Complementary and Alternative Medicine, vol. 12, p. 73, 2012.

[23] P. E. Shrout and J. L. Fleiss, "Intraclass correlations: uses in assessing rater reliability," Psychological Bulletin, vol. 86, no. 2, pp. 420-428, 1979.

[24] D. V. Cicchetti and T. Allison, "A new procedure for assessing reliability of scoring EEG sleep recordings," American Journal of EEG Technology, vol. 11, no. 3, pp. 101-110, 1971.

[25] K. F. Leung, F. B. Liu, L. Zhao, J. Q. Fang, K. Chan, and L. Z. Lin, "Development and validation of the Chinese quality of life instrument," Health and Quality of Life Outcomes, vol. 3, p. 26, 2005.

[26] J. L. Fleiss, The Design and Analysis of Clinical Experiments, John Wiley and Sons, New York, NY, USA, 1986.

[27] Y. Y. Zhao, X. Z. Lin, M. Z. Zhang, X. Zou, and H. L. Wu, "Professor Deng Tietao's experience in diagnosis and treatment of coronary heart disease based on the theory of heart and spleen," Journal of New Chinese Medicine, vol. 39, no. 4, pp. 5-6, 2007.

[28] J. C. Hobart, S. J. Cano, T. T. Warner, and A. J. Thompson, "What sample sizes for reliability and validity studies in neurology?" Journal of Neurology, vol. 259, no. 12, pp. 2681-2694, 2012. 